\title{
MEJORAMIENTO DEL RENDIMIENTO Y LA PRECOCIDAD DEL FRIJOL (Phaseolus vulgaris L.) ${ }^{1}$
}

\author{
Rafael Rodríguez C., Carlos Orellana ${ }^{2}$
}

\section{COMPENDIO}

Tomando en cuenta la complejidad del carácter rendimiento y las ventajas que la precocidad presenta para el agricultor, se Inició el presente trabajo en 1985, con el objeto de encontrar genotipos con un grado de precocidad aceptable y que a la vez conserven el rendimiento. Asimismo, se buscaron materiales altamente rendidores, aunque no necesariamente precoces. Se empleó el Método Masivo, buscando avanzar hasta la generación $\mathrm{F}_{6}, 25$ poblaciones provenientes de cruzamientos entre diez progenitores con diversa duración de ciclo. En $\mathrm{F}_{6}$ se realizaron 458 selecciones individuales, considerando los caracteres: Rendimiento por planta, precocidad y una buena visual de los mismos. En $\mathrm{F}_{7}$, se realizó la prueba de progenies para comprobar el éxito de la selección en F6, En cuanto a rendimiento se observó una disminución del mismo, explicable en cuanto al manejo diferente dado a las selecciones individuales $\mathrm{F}_{6}$ comparado con las progenies $\mathrm{F}_{7}$. En cuanto a precocidad, se observaron algunas progenies bastante aceptables y con un rendimiento que no es usual en materiales de ciclo corto, bajo condiciones del Suroriente de Guatemala (superiores a 1,5 toneladas por hectárea).

\section{INTRODUCCIÓN}

Existen dos factores que a corto plazo determinarán en el área centroamericana y el Caribe, el avance en productividad debido a mejoramiento genético en el cultivo del frijol, son el potencial de rendimiento y la resistencia a enfermedades y plagas.

El rendimiento ha sido clasificado como un carácter controlado por herencia cuantitativa. Es el resultado de la acción de muchos genes de efectos menores en su mayoría no identificados, todos contribuyendo al rendimiento total o interaccionando fuertemente con el ambiente. Por aparte, la precocidad presenta ventajas tales como: a) Ayuda a escapar a factores adversos del medio, b) Obtener alimento en un menor período de tiempo, c) Lograr una comercialización o ventade1granoa más corto plazo, d) Reducir entre 10 y 15 días el trabajo de campo, e) Facilitar rotaciones de cultivos en el campo, y f) Reducir los riesgos por exposición prolongada del cultivo a factores adversos del medio.

Sin embargo, la precocidad impone ciertas limitaciones sobre la capacidad biológica del cultivo, ya que un ciclo corto limita la fotosíntesis total y hasta cierto punto, el potencial de rendimiento. Adicionalmente, se

\begin{abstract}
${ }^{3}$
Because of the complexity that yield components have, and the advantages that early varieties have for farmers considering actual yield components, genotype evaluation was initiated in 1985 to Identify early maturity types. At the same time, high yielding varieties were evaluated that were not necessarily early. The bulk selection method was used to advance to the F6 generation. Twenty rive populations from crosses among 10 parents with diverse maturities were evaluated. At $\mathrm{F}_{6}, 458$ individual selections were made considering yield per plant, early maturity and visual characteristics. At $F_{7}$ aprogeny test was made to evaluate $F_{6}$ effectiveness Grain yields were reduced which is in part explained by the management given to the individual $\mathrm{F}_{6}$ selections compared to $\mathrm{F}_{7}$. Regarding early maturity, various were found with acceptable grain yields which are not common amongst early maturity varieties in the southeastern region of Guatemala (above $1-5 \mathrm{Mg} / \mathrm{ha}$ ).
\end{abstract}

observa una reducción en la biomasa total de la planta, lo cual incide en un menor número de yemas florales, y por tanto menor rendimiento de grano.

Debido a que el ciclo se acorta, al completar la planta su ciclo vegetativo presenta una arquitectura pobre en cuanto a altura, vigor, ramificación, etc. y, en consecuencia, menor número de puntos florales productivos.

\section{OBJETIVOS}

Considerando lo anterior, se planificó el presente estudio con el objeto de encontrar genotipos con un grado de precocidad aceptable (promedio de 60 días a madurez fisiológica) y que a la vez conserven el rendimiento. De igual manera se espera encontrar buenas combinaciones entre genotipos rendidores, aunque no presenten una precocidad aceptable.

${ }_{1}^{1}$ Presentado en la XXXV Reunión Anual del PCCMCA, San Pedro Sula, Honduras, 1989.

${ }^{2}$ Profesional II y Técnico Profesional III, respectivamente, Programa de Frijol, ICTA-Guatemala.

${ }^{3} \mathrm{El}$ Abstract es traducción del Compendio. Elaborado por el Comité Editorial para mostrar el formato de presentación de los artículos.

${ }^{1}$ Publicado en Agronomía Mesoamericana Vol. 1 (1990). 


\section{HIPÓTESIS}

Sobre la base de que si la selección se practica en generaciones avanzadas, la posibilidad de mayor éxito en la misma aumenta, se ha considerado en este trabajo emplear el Método Masivo, con el fin de avanzar las poblaciones segregantes en estudio hasta la generación $\mathrm{F}_{6}$, esperando con esto contar con individuos altamente homocigotos para el carácter a seleccionar, en este caso, rendimiento, y evitar en alguna medida con esto, segregaciones indeseables o mermas en el rendimiento en generaciones avanzadas, cuando la selección se ha practicado en generaciones tempranas.

\section{MATERIALES Y MÉTODOS}

\section{Localización}

La primera fase de este trabajo (Cruzamientos, $\mathrm{F}_{1}$ y $\mathrm{F}_{2}$ ) se realizó en el Centro Internacional de Agricultura Tropical, CIAT, en Cali, Colombia. El manejo, identificación y detalles en general de esta fase aparecen en la Memoria Anual del Programa de Frijol, 1988.

La prueba de progenies $\mathrm{F}_{7}$, que se reporta en este trabajo se llevó acabo en las instalaciones del Centro de Producción Agrícola de Oriente del ICTA, Aldea Río de la Virgen, Jutiapa, Guatemala.

\section{Duración}

Bajo las condiciones del Suroriente de Guatemala, el trabajo ha seguido la siguiente cronología:

\section{Poblaciones F3: Septiembre - noviembre 1986 \\ F4: Enero - abril 1987 \\ F5: Mayo - agosto 1987 \\ F6: Septiembre - noviembre 1987}

La prueba de progenies $\mathrm{F}_{7}$, se sembró el 7 de junio de 1988 y se cosechó en septiembre del mismo año.

\section{Tratamientos y Diseño Experimental}

Se evaluaron 458 progenies $\mathrm{F}_{7}$, a surco por selección individual realizada en 176. Todas las progenies provenientes de una misma población se agruparon, para luego colocar a los respectivos progenitores contiguamente.

El largo del surco fue de $3 \mathrm{~m}$ para cosechar 2,5 m. Se utilizó la densidad de siembra comercial recomendada en la región (250.000 plantas por hectárea) resultante de separar los surcos a $0,40 \mathrm{~m}$ y colocar una semilla a cada $0,10 \mathrm{~m}$. No se utilizó ningún diseño experimental.

\section{Manejo Experimental}

El suelo fue arado, rastreado y surqueado con tractor. Al momento de la siembra se fertilizó con 20-20-0, mezcla química en una dosis de $194 \mathrm{~kg} / \mathrm{ha}$ y se desinfestó con Phoxim (Volatón 5G) a razón de $45 \mathrm{~kg} / \mathrm{ha}$, ambos colocados al fondo del surco.

Los controles fitosanitarios se realizaron en forma preventiva y oportuna, tanto de plagas como de enfermedades, con el fin de observar la expresión de rendimiento y de precocidad en las progenies.

\section{Variables de Respuesta}

Días a Floración: Contados desde la colocación de la semilla en suelo húmedo, hasta que el $50 \%$ de las plantas sobre la parcela neta mostraron, por lo menos, una flor abierta.

Días a madurez fisiológica: Contados desde la siembra en suelo húmedo hasta que el $90 \%$ de las vainas sobre la parcela neta cambiaron su color verde al típico de la progenie.

Días a cosecha: Contados desde la siembra en suelo húmedo hasta obtener características de secamiento óptimo para la trilla.

Período de llenado de grano: Los días contados entre la floración y la madurez fisiológica.

Rendimiento: Expresado en $\mathrm{kg} / \mathrm{ha}$ y corregido al $14 \%$ de humedad.

Rendimiento por Día: Resultante de dividir el rendimiento de grano entre los días a madurez fisiológica.

Rendimiento por Planta: Resultante de dividir el rendimiento entre el número de plantas cosechadas sobre la parcela neta.

\section{Análisis de la Información}

En función de las variables estudiadas, se separaron las progenies que mostraron 60 o menos días a madurez y con un rendimiento aceptable en un grupo y las más rendidoras en otro, seleccionando posteriormente en cada grupo, las 5 mejores progenies.

Debido a que sólo se empleó una repetición, no fue posible hacer promedios, por lo que por inspección y comparación, se realizaron las selecciones mencionadas.

\section{Criterios de Selección}

Para el grupo precoz se aplicó un criterio de 60 o menos días a madurez fisiológica, combinado con un rendimiento de grano mínimo de 140 gramos por metro cuadrado, necesario para poder realizar la corrección al $14 \%$ de humedad.

Para el grupo rendidor, el criterio de selección empleado fue el de superar los $250 \mathrm{~g} / \mathrm{m}^{2}$ de rendimiento de grano. 


\section{RESULTADOS Y DISCUSIÓN}

En el Cuadro 1 puede observarse el grupo seleccionado como precoz. Este representa una presión de selección de $3,71 \%$.

En los objetivos del trabajo se mencionó la búsqueda de genotipos precoces que logren conservar un rendimiento aceptable como lo han sugerido Quiñónez (1965) y Rodríguez et al. (1981).

Se hace necesario entonces recordar la relación negativa que han encontrado varios autores entre precocidad y alto rendimiento, tales como Laing et al. (1983), Leiva (1977), Ajquejay y Masaya (1980) y Rodríguez (1986).

Sin embargo, debido a que en el estudio se incluyeron tanto progenitores precoces como rendidores, las progenies resultantes de las diferentes combinaciones efectuadas pudieron mostrar una buena variabilidad para los caracteres precocidad, rendimiento y una buena combinación de ambos.

Lo anterior puede verse claramente en el Cuadro 1, en donde destacan progenies como la 24-14, que con 57 días a madurez expresó el mayor rendimiento de grano de todo el grupo precoz y adicionalmente mostró uno de los más altos períodos de llenado de grano, relación positiva entre período de llenado de grano y rendimiento, que concuerda con lo encontrado por Izquierdo y Hosfield (1983) y Rodríguez (1986).

Todo lo anterior se mantiene para las restantes progenies que conforman el grupo élite seleccionado de 5 materiales, estos son: 25-4, 19-31, 4-3 y 24-2.

$\mathrm{Al}$ realizar una corrección por los días a madurez, traducido en rendimiento diario, se observa un material,
24-14, que presenta el mayor valor de todo el grupo para esta variable y que además es comparable al que muestran varias progenies seleccionadas dentro del grupo rendidor, como se verá más adelante.

Para este grupo élite intervienen 7 de los 10 progenitores empleados (no intervienen BAT 304, Negro Huasteco 81 y A 321), como puede comprobarse observando el Cuadro 2.

Al analizar el grupo rendidor, puede verse en el Cuadro 3, que con una presión de selección de 3,93\% la progenie con mayor rendimiento de grano fue la 16-12, que al mismo tiempo muestra días a madurez bastante aceptables.

De igual forma presenta el rendimiento diario más alto del grupo.

Seleccionando las 5 mejores progenies del grupo, por alto rendimiento, se pueden adicionar las progenies $16-21,16,5,16-11$ y $11-9$.

Nuevamente, se vuelve a encontrar una buena relación entre el alto rendimiento Y un largo período de llenado de grano.

Los días a madurez fisiológica pueden considerarse intermedios para este grupo élite.

En $\mathrm{F}_{6}$, se considera que la homocigosis ha alcanzado aproximadamente $97 \%$, razón por la cual se esperaba un cambio no muy drástico en rendimiento por planta al pasar a $\mathrm{F}_{7}$. Sin embargo, esto es notorio en el Cuadro 3, en donde, en general, a excepción de contados casos, en donde los valores se mantienen, el rendimiento por planta en $\mathrm{F}_{6}$ se reduce en alrededor de un $50 \%$ al pasar a $\mathrm{F}_{7}$.

Cuadro 1. Progenies $\mathrm{F}_{7}$ precoces ( 60 a menos días a madurez) provenientes de 25 poblaciones $\mathrm{F}_{6}$ Jutiapa, junio-setiembre, 1988.

\begin{tabular}{llcccccc}
\hline Población & Genealogía & Selección & $\begin{array}{c}\text { Días a } \\
\text { madurez }\end{array}$ & $\begin{array}{c}\text { Días a } \\
\text { cosecha }\end{array}$ & $\begin{array}{c}\text { Pllga } \\
\text { (días) }\end{array}$ & $\begin{array}{c}\text { Rend. } \\
\text { (kg/ha) } \\
(\mathbf{1 4 \%})\end{array}$ & $\begin{array}{c}\text { Rend/Día } \\
\text { (kg/ha) } \\
(\mathbf{1 4 \%})\end{array}$ \\
\hline \multirow{2}{*}{ DR 8299 } & Negro Huasteco 81 x BAT 304 & $1-1$ & 60 & 73 & 31 & 1.522 & 25,37 \\
NXDC, 11016 & ICTA-Quetzal X XAN 112 & $4-3$ & 60 & 72 & 30 & 1.608 & 26,80 \\
NTDG 3344 & ICTA-Querzal x Huetar & $6-1$ & 60 & 73 & 28 & 1.379 & 22,98 \\
& & $6-7$ & 52 & 60 & 26 & 1.130 & 21,73 \\
& & $6-10$ & 54 & 62 & 29 & 1.380 & 25,55 \\
NPDG 13350 & BAT 304 x Ju 84-3 & $12-3$ & 60 & 73 & 30 & 1.446 & 24,10 \\
NPDG 13353 & XAN 112 x Ju 84-3 & $15-3$ & 60 & 74 & 28 & 1.545 & 25,75 \\
& & $15-16$ & 58 & 65 & 28 & 1.368 & 23,41 \\
NPDG 13356 & \multirow{2}{*}{ Ju 84-3 x Pata de Zope } & $18-12$ & 60 & 74 & 30 & 1.558 & 25,97 \\
NPDG 13357 & Ju 84-7 x Huetar & $19-10$ & 60 & 75 & 28 & 1.533 & 25,88 \\
& & $19-21$ & 59 & 75 & 28 & 1.369 & 23,20 \\
NPDG 13386 & \multirow{2}{*}{ Ju 84-3 x Rabia de Gato } & $19-31$ & 60 & 70 & 28 & 1.809 & 30,15 \\
& & $24-2$ & 56 & 65 & 30 & 1.592 & 28,43 \\
& & $24-5$ & 56 & 72 & 28 & 1.544 & 27,57 \\
NPDG 13387 & Ju 84-7 x Pata de Zope t, & $24-14$ & 57 & 72 & 30 & 2.428 & 42,60 \\
& & $25-4$ & 60 & 67 & 32 & 1.886 & 31,43 \\
& & $25-6$ & 59 & 67 & 31 & 1.552 & 26,30 \\
\hline
\end{tabular}

a: Período de llenado de grano. 
Cuadro 2. Datos de los progenitores empleados en el estudio, provenientes de la misma época de evaluación de las progenies $\mathrm{F}_{7}$, Iutiapa, junio-septiembre, 1988.

\begin{tabular}{lcccccc}
\hline Progenitor & $\begin{array}{c}\text { Carácter } \\
\text { Madurez }\end{array}$ & $\begin{array}{c}\text { Días a } \\
\text { Cosecha }\end{array}$ & $\begin{array}{c}\text { Días } \\
\text { (días) }\end{array}$ & $\begin{array}{c}\text { Plga } \\
(\mathbf{1 4 \% )})\end{array}$ & $\begin{array}{c}\text { Rend. } \\
\mathbf{( k g / h a )} \\
\text { (kg/ha) }\end{array}$ & $\begin{array}{c}\text { Rend. } \\
\text { por día }\end{array}$ \\
\hline Rabia de Gato & Precocidad & 57 & 66 & 31 & 1457 & 25.56 \\
Pata de Zope & “ & 58 & 65 & 30 & 1707 & 29.43 \\
Huetar & “ & 57 & 72 & 27 & 1780 & 31.22 \\
Ju 84-3 & “ & 60 & 76 & 30 & 1221 & 20.35 \\
Ju 84-7 & “ & 62 & 72 & 29 & 1826 & 29.45 \\
RAT 304 & Rendimiento & 60 & 72 & 28 & 1227 & 20.45 \\
XAN 112 & “ & 67 & 77 & 33 & 1972 & 29.43 \\
Negro Huasteco-81 & “ & 70 & 79 & 35 & 1739 & 24.84 \\
ICTA-Quetzal & “ & 68 & 78 & 33 & 1499 & 22.04 \\
A321 & & - & - & - & - & - \\
\hline
\end{tabular}

a: Período de llenado de grano

b: Se mostró completamente desadaptado

Cuadro 3. Progenies $F_{7}$ de alto rendimiento provenientes de 25 poblaciones $F_{6}$ Jutiapa, junio-septiembre, 1988.

\begin{tabular}{|c|c|c|c|c|c|c|c|c|c|}
\hline Población & Genealogía & Selección & $\begin{array}{c}\text { Días a } \\
\text { Madurez }\end{array}$ & $\begin{array}{c}\text { Días a } \\
\text { Cosecha }\end{array}$ & $\begin{array}{l}\text { PIIga } \\
\text { (días) }\end{array}$ & $\begin{array}{l}\text { Rend. } \\
\text { kg/ha) } \\
(14 \%)\end{array}$ & $\begin{array}{l}\text { Rend. } \\
\text { por día } \\
\text { (kg/ha) }\end{array}$ & $\begin{array}{l}\text { Rend. } \\
\text { planta } \\
\text { F }_{6}(\mathrm{~g})\end{array}$ & $\begin{array}{c}\text { Rend. } \\
\text { por planta } \\
\mathbf{F}_{7}(\mathbf{g})\end{array}$ \\
\hline DR 8299 & Negro Husteco 81 X BAT 304 & $1-18$ & 70 & 76 & 34 & 2.612 & 37,31 & 21 & 10 \\
\hline NTI)G 13346 & ICTA-Quetzal x Ju 84-3 & $8-6$ & 74 & so & 37 & 2.450 & 33,11 & 24 & 12 \\
\hline \multirow[t]{4}{*}{ NPDG 13347} & Negro Husteco 81 x A321 & $9-1$ & 63 & 73 & 30 & 2.724 & 43,24 & 15 & 11 \\
\hline & & $9-13$ & 67 & 78 & 33 & 2.717 & 40,55 & 18 & 12 \\
\hline & & $9-18$ & 69 & 76 & 34 & 2.789 & 40,42 & 32 & 12 \\
\hline & & $9-21$ & 67 & 74 & 35 & 2.488 & 37,13 & 15 & 12 \\
\hline \multirow[t]{2}{*}{ NPTD 13349} & BAT 304 x A 321 & $11-9$ & 73 & 83 & 39 & 2.846 & 38,99 & 12 & 11 \\
\hline & & $11-17$ & 73 & 77 & 35 & 2.513 & 34,42 & 27 & 10 \\
\hline \multirow[t]{8}{*}{ NPDCY 13354} & A $321 \times$ Ju $84-3$ & $16-4$ & 69 & 76 & 37 & 2.706 & 39,22 & 15 & 11 \\
\hline & & $16-5$ & 74 & 82 & 40 & 2.924 & 39,51 & 28 & 13 \\
\hline & & $16-11$ & 65 & 74 & 36 & 2.896 & 44,55 & 24 & 12 \\
\hline & & $16-12$ & 63 & 76 & 33 & 3.354 & 53,24 & 14 & 15 \\
\hline & & $16-13$ & 63 & 74 & 31 & 2.500 & 39,68 & 16 & lo \\
\hline & & $16-15$ & 69 & 76 & 35 & 2.645 & 38,33 & 21 & 13 \\
\hline & & $16-16$ & 68 & 79 & 35 & 2.690 & 39,56 & 27 & 13 \\
\hline & & $16-21$ & 69 & 79 & 36 & 2.936 & 42,55 & 22 & 14 \\
\hline \multirow[t]{2}{*}{ NPTG 13358} & Huetar x Pata de Zope & $20-18$ & 63 & 77 & 32 & 2.648 & 42,03 & 12 & 11 \\
\hline & & $20-24$ & 67 & 78 & 32 & 2.562 & 38,24 & 13 & 11 \\
\hline
\end{tabular}

a: Período de llenado de grano.

Lo anterior podría tener explicación en el hecho de que el manejo dado a las selecciones individuales en $\mathrm{F}_{6}$ no fue el mismo dado a las progenies $\mathrm{F}_{7}$, principalmente en cuanto a corrección a $14 \%$ de humedad del grano, que en $\mathrm{F}_{6}$ no se hizo y si en $\mathrm{F}_{7}$. Luego, la época de selección no fue la misma que la de comprobación de progenies, es decir, $\mathrm{F}_{6}$ se hizo en setiembre-noviembre de 1987 y F $_{7}$ en junio-septiembre de 1988. Aunque ambas se consideran buenas épocas para la siembra de frijol en la región, la $\mathrm{F}_{6}$ hubo necesidad de auxiliarla con riego (ver climograma para Jutiapa, Memoria Anual, Programa de Frijol, 1987), mientras que para $F_{2}$, como se muestra en el climograma para Jutiapa, 1988 de esta Memoria Anual, se notaron dos bajas en la precipitación muy acentuadas, que pudieron haber afectado el rendimiento de grano final.

Para el grupo élite (5 progenies) de alto rendimiento, intervienen solamente 3 de los 10 progenitores seleccionados, siendo éstos BAT 304, A 321 y Ju 84-3.

Es importante, también, señalar el buen comportamiento como progenitor que muestra Ju 84-3 al intervenir como tal en progenies tanto precoces como rendidoras, lo cual refleja una buena habilidad para combinarse con otros materiales y generar progenies ventajosas. 
Finalmente, es interesante notar las progenies 20-18 y 20-24 del Cuadro 3, cuyos progenitores son dos variedades muy precoces, las cuales están generando dos progenies que fueron seleccionadas por alto rendimiento, lo cual, como ya se mencionó antes, existen evidencias a favor y en contra del carácter positivo o negativo de la relación precocidad-rendimiento.

\section{CONCLUSIONES}

1. En todas las consideraciones hechas en este trabajo, siempre se mantuvo el énfasis en grano negro, preferido por el consumidor final, a quien se dirigen los beneficios de la investigación.

2. La disminución de rendimiento por planta al pasar de $\mathrm{F}_{6}$ a $\mathrm{F}_{7}$ probablemente como consecuencia de la corrección por humedad al 14\% realizada en $\mathrm{F}_{7} \mathrm{y}$, que no se hizo en $\mathrm{F}_{6}$. Asimismo, la época para ambas generaciones fue diferente.

3. Las dos generaciones estudiadas fueron manejadas a la densidad comercial recomendada en la región $\mathrm{y}$ las selecciones individuales en $\mathrm{F}_{6}$, se realizaron bajo competencia completa.

4. De acuerdo con los resultados obtenidos, se pudo observar que es factible unir una precocidad aceptable con un rendimiento aceptable, lo que contradice la relación negativa encontrada por algunos autores. Es probable que lo anterior esté influenciado por el hecho de tratarse, en este caso, de un gran número de progenies con gran variabilidad entre ellas para los caracteres buscados, y no de variedades definidas.

El período de llenado de grano se le encuentra siempre relacionado a buen rendimiento, sin importar si la progenie es precoz, intermedia o tardía.

Se identificó una línea, Ju 84-3 que muestra buenas características como progenitor, al combinarse con otro, tanto para precocidad como para rendimiento,

En cuanto a precocidad, se observaron algunas progenies bastante aceptables y con un rendimiento que no es usual en materiales de ciclo corto, bajo condiciones del Suroriente de Guatemala.

\section{RECOMENDACIÓN}

Realizar pruebas preliminares de rendimiento bajo diseño experimental y en localidades.

\section{BIBLIOGRAFIA}

QUEJAY A., S; MASAYA S., P. 1980. Influencia de la densidad y la fertilización en seis genotipos de frijol (Phaseolus vulgaris L.) en dos zonas frijoleras de Guatemala. Instituto de Ciencia y Tecnología Agrícolas. In Reunión Anual del PCCMCA (26, 1980, Guatemala) Guatemala. 21 p.

INSTITUTO DE CIENCIA Y TECNOLOGIA AGRICOLA. 1987. Memoria Anual, Programa de Frijol, 1986-87. 105 p.

1988. Memoria Anual, Programa de Frijol, 1987-88. 148 p.

IZQUIERDO, J.A.; HOSFIELD, G.L. 1983. The relationship of seed filling to yield among dry beans with differing architectural forms. J. Amer. Soc. Hort. Sci. 108:106.

LAING, D.R.; KRETCHMER, PJ.; ZULUAGA, S.; JONES, P.G. 1983. Field bean. In Smith, W.H. and SJ. Banta, ed. Symposium on Potential Productivity of field crops under Different Environments. Los Baños, Philippines, 1980. (Proceedings). Manila, International Rice Research Institute. p. 227-248.

LEIVA R., O. R. 1977. Herencia y mejoramiento de la precocidad del frijol (Phaseolus vulgaris L.) en el trópico. Tesis Mag. Sc. Bogotá, Universidad Nacional de Colombia-Instituto Colombiano Agropecuario. 80 p.

QUIÑNONEZ, F. E. 1965. Correlation of characters in dry beans. Proc. Amer. Soc. Hort Sci. 86:368-372.

RODRIGUEZ G., R. R. 1986. Caracterización morfofisiológica e identificación de caracteres para mayor rendimiento en genotipos precoces de frijol común (Phaseolus vulgaris L.) Tesis de Maestría en Ciencias. Colegio de Postgraduados. Chapingo, México. 178 p.

RODRIGUEZ DE LEON, C.A.; ALDANA DE LEON, L.F.; MASAYA S., P. 1981. Caracterizaci6n de variedades de frijol (Phaseolus vulgaris L.) usadas en el Sur-Oriente de Guatemala. In Reunión Anual del PCCMCA (27,198 1, Santo Domingo, República Dominicana). Santo Domingo, República Dominicana. p. L-1-1-L1-11. 